\section{ORIGINAL RESEARCH}

P.D. Griffiths

M.J. Reeves

J.E. Morris

G. Mason

S.A. Russell

M.N.J. Paley

E.H. Whitby

\title{
A Prospective Study of Fetuses with Isolated Ventriculomegaly Investigated by Antenatal Sonography and In Utero MR Imaging
}

\begin{abstract}
BACKGROUND AND PURPOSE: Fetal ventriculomegaly (VM) is important because of its high prevalence and high risk of association with other brain abnormalities. The purpose of this article was to investigate the hypotheses that including in utero MR imaging (iuMR) in the diagnostic pathway for fetuses with isolated VM on antenatal imaging will show other brain abnormalities in a high proportion of cases and that these will have a significant effect on clinical management.
\end{abstract}

MATERIALS AND METHODS: One hundred forty-seven pregnant women were recruited prospectively from 8 fetomaternal centers in Britain. All of the fetuses had VM diagnosed on sonography but no other abnormality. iuMR was performed, and the results of the examinations were compared with those of sonography. Two fetomaternal experts made independent assessments of the effects of any new diagnoses on clinical management.

RESULTS: Categoric assessments of ventricular size were the same in approximately $90 \%$ of fetuses. Other abnormalities were shown in $17 \%$ of fetuses. The most frequent additional brain abnormality shown on iuMR was agenesis of the corpus callosum. Severe VM was associated with an approximately 10 -fold increase in the risk of another brain abnormality being present when compared with fetuses with mild VM. The most profound effects on clinical management, however, were found in cases of mild VM.

CONCLUSIONS: This work supports our hypotheses by showing a high detection rate of other brain pathology when iuMR was used to supplement antenatal sonography (17\%). In a high proportion of cases, the detection of the extra pathology would have led to significant changes in clinical management.

$\mathbf{S}_{\mathrm{n}}^{\mathrm{n}}$ tructural abnormalities of the fetus are investigated in many countries by using sonography, first by way of screening programs and then by detailed anomaly scanning if necessary. It is desirable to have as much anatomic information available as possible for the parents for 2 main reasons: First, finding no abnormality is of great comfort to parents, particularly if the fetus is at increased risk of malformation. Second, if abnormalities are found, the parents need as much accurate information as possible to assist in making decisions about the future of the pregnancy.

Abnormalities of the brain and spine are among the most common causes for concern in fetal imaging, and ventriculomegaly (VM) is one of the most frequent abnormal brain find-

Received April 1, 2009; accepted after revision June 3.

From the Academic Unit of Radiology (P.D.G., M.J.R., J.E.M., M.N.J.P., E.H.W.), University of Sheffield, Sheffield, UK; Department of Feto-Maternal Medicine (G.M.), Leeds General Infirmary, Leeds, UK; and Radiology Department (S.A.R.), St. Mary's Hospital, Manchester, UK.

We are grateful for the support of the Wellcome Trust, UK. We would also like to acknowledge Westfield Health for financial support for the salary of Mike Reeves, MD, as a Clinical Research Fellow. Elspeth Whitby, MD, was supported by The Health Foundation via the Academy of Medical Sciences. Westfield Health and The Health Foundation did not have any role in the design or conduction of this study, or in the preparation of this manuscript.

The study was approved by the South Sheffield Research Ethics Committee and sponsored by the Wellcome Trust, UK.

Please address correspondence to Paul Griffiths, MD, Academic Unit of Radiology, C Floor, Royal Hallamshire Hospital, Glossop Rd, Sheffield S10 2JF, United Kingdom; e-mail: p.griffiths@sheffield.ac.uk

Indicates article with supplemental on-line tables.

Indicates open access to non-subscribers at www.ajnr.org

DOI 10.3174/ajnr.A1767 ings. The widely used definition of fetal VM is a transtrigone measurement of $\geq 10 \mathrm{~mm}$ at any stage of pregnancy. ${ }^{1-3}$ With that definition, VM is found in $\leq 2.5$ per 1000 pregnancies. ${ }^{4}$ In some cases, VM is the only abnormal finding, leading to the term "isolated VM," which is found in approximately $20 \%$ of all cases of fetal VM diagnosed on sonography (eg, 22\% from 267 cases $^{5}$ and $16 \%$ from 51 cases $^{6}$ ). Fetuses with isolated VM are at an increased risk of aneuploidy, ${ }^{7}$ particularly trisomy 21 , and amniocentesis is often offered for this reason.

It is important to recognize VM antenatally because it may be an indicator and/or manifestation of other serious central nervous system (CNS) abnormalities with values of $88 \%$ sensitivity in 1 study. ${ }^{8}$ Fetal VM is associated with poor outcome in terms of both mortality and morbidity if projected outcome data from termination of pregnancy cases are included. ${ }^{9}$ Once VM has been demonstrated on a screening sonography examination, women are referred to specialist fetal/maternal centers for further sonographic assessment, which includes detailed neurosonography by an expert in fetal imaging. The implications of detecting fetal VM are complicated because there are many underlying etiologies. Most authorities believe that if other structural CNS abnormalities are found in conjunction with VM, there is a high risk of a poor neurologic and/or developmental outcome. When VM is the only abnormal finding and the fetus is known to be euploid, counseling parents is partly based on the severity of the VM because increasing size of the ventricles is associated with a higher risk of poor outcome. The most recent data on outcome have used the results of in utero MR imaging (iuMR) to define isolated VM. ${ }^{10}$

The purpose of this study was to investigate the hypotheses 
that including iuMR in the diagnostic pathway for fetuses with isolated VM on antenatal imaging will show other brain abnormalities in a high proportion of cases and that these will have a significant effect on clinical management. We have attempted to quantify these potential benefits to management.

\section{Materials and Methods}

The study was approved by the South Sheffield Research Ethics Committee and sponsored by the Wellcome Trust, United Kingdom. None of the cases have been previously reported in our earlier publications on diagnostic accuracy assessment on iuMR.

\section{Participants}

One hundred forty-seven pregnant women were recruited from 8 tertiary fetal assessment units in England and Scotland. Entrance criteria for the study were the following: singleton pregnancy with fetal $\mathrm{VM}$ diagnosed on sonography (trigone measurement of $\geq 10 \mathrm{~mm}$ ) referred to the study center at 20 weeks' gestational age or later and no other abnormality of the fetus shown by sonography (brain or somatic) under good sonographic conditions (eg, no obscuration because of maternal body mass index problems, fetal position, or oligohydramnios). None of the fetuses had a known abnormality on karyotyping at the time of referral. The women did not have any known or suspected contraindications to MR imaging and agreed to provide written consent after full explanation by 1 of the authors. The women were not paid for their involvement in the study, but travel expenses were offered for themselves and a companion. Most iuMR examinations were performed within 4 days of the referral; exceptions included periods of machine breakdown and the referral being made before 20 weeks' gestational age (in which case the examination was delayed to 20-21 weeks' gestation). The study extended from 2005 to 2009.

\section{Procedures}

Our iuMR technique has been described in detail elsewhere ${ }^{11}$ but is summarized here. All images were acquired on a 1.5T scanner (either Infineon, Philips Medical Systems, Best, the Netherlands; or HDx, GE Healthcare, Milwaukee, Wisconsin). A flexible phased array body coil was attached around the lower abdomen, and a series of 3-plane scout views was obtained. Once the fetal head was located, single-shot fast spin-echo sequences were run by using the following typical parameters: TR, 20,000 ms; $\mathrm{TE}_{\text {eff }}, 75 \mathrm{~ms}$; echo-train length, 132; FOV, 25 $\mathrm{cm}$; matrix size, $248 \times 256$; NEX, 1 ; FA, $120^{\circ}$. Twenty 5-mm-thick sections of the fetal brain were obtained (20-second acquisitions) in the 3 natural orthogonal planes. After these were judged to be of diagnostic quality, similar acquisitions were performed by using 3-mm-thick sections with TR, 31,416 ms; $\mathrm{TE}_{\mathrm{eff}}$, $92 \mathrm{~ms}$; echo-train length, 136; FOV, $25 \mathrm{~cm}$; matrix size, $183 \times 256$; NEX, 1 ; refocusing angle, $120^{\circ}$. An axial gradient-echo T1-weighted sequence was acquired in 30-second acquisition time, either with or without suspended maternal respiration. The parameters for the T1 sequence were the following: TR, $238 \mathrm{~ms}$; TE, $3.4 \mathrm{~ms}$; refocusing angle, $70^{\circ}$; FOV, $23 \mathrm{~cm}$; matrix size, $192 \times 256$; NEX, 1 .

The iuMR images were reported for clinical purposes at the time of the examination but, for the purposes of this study, were later reviewed by a neuroradiologist experienced in iuMR brain studies (P.D.G.). The reviewer had access to the sonography report at the time of review but to no subsequent follow-up information. The size of the trigones of the lateral ventricles was determined on sonography on a standardized axial view by the referring fetomaternal expert. A similar assessment of the transverse diameter of the trigones of both lateral ventricles was performed on the axial MR images by 1 of 2 experienced operators (J.E.M. or M.J.R.). This was done in a blinded fashion-that is, the reviewers did not know the trigone measurements made on sonography when they made the measurements on the iuMR studies. The measurements made on sonography were compared with those obtained from iuMR on the basis of any change in categoric description: mild (10-12 mm), moderate (13-15 mm), and severe $(\geq 16 \mathrm{~mm}$ ) based on the size of the largest of the ventricles if asymmetric. The final categoric assignment was made on the basis of the MR imaging measurements. Any other brain abnormalities were recorded, and the results of the iuMR examination were passed on to the referring clinician. The pick-up rate of brain pathology other than VM on iuMR was taken to be an indicator of improved diagnostic accuracy when compared with sonography. The gestational age of the fetus at the time of referral for iuMR was used to divide the patients into 2 groups for further analysis: namely, those referred at $20-24$ weeks' gestational age (20-24 weeks' group) and those referred at 25 weeks or after ( $25+$ weeks; group).

We did not study the clinical significance of the results of iuMR in our patients in real-time; instead we made a retrospective assessment of possible effects on clinical management. This was performed by presenting the results of the sonographic and iuMR studies for each patient to 2 fetomaternal experts independently (G.M., S.A.R.). Each expert reported the change in management that would have been brought about by including iuMR in the diagnostic pathway, in their opinion. One of 4 categoric responses was used to define these data as described in the footnote of on-line Table 1, and their results are presented in on-line Tables 1 and 2.

Results for diagnostic agreement and the proportion of cases in which additional diagnostic information was found are expressed as percentages, with $95 \%$ confidence intervals (CI) estimated by using the binomial method.

\section{Results}

One hundred forty-seven consecutive singleton fetuses with apparent isolated VM were recruited, of which 99 were referred between 20-24 weeks and the remaining 48 referred at $\geq 25$ weeks' gestational age. Overall, the diagnosis of isolated VM was supported by iuMR in 122/147 (83\%; 95\% CI, 76\%$89 \%$ ) cases, while other brain abnormalities were shown on iuMR in 25/147 (17\%). In the 122 cases of confirmed isolated $\mathrm{VM}$, the categoric assessments of the largest trigone size made on sonography were compared with those made on iuMR. There was complete categoric agreement in $90 \%$ of cases $(95 \%$ CI, 83\%-95\%). In the 12 cases with categoric disagreements, 2 fetuses were reclassified from mild VM on sonography to normal-sized ventricles on iuMR, 9 fetuses were reclassified from mild VM on sonography to moderate VM on iuMR, and 1 , from moderate on sonography to severe on iuMR.

\section{Twenty to 24 Weeks' Group}

The median gestational age at the time of iuMR in this group was 22 weeks. Additional brain pathology was identified in 9/99 (9\%; 95\% CI, 4\%-17\%) fetuses imaged between 20 and 24 weeks' gestational age. The categories of enlarged trigones (on the most severely affected side) in the 90 confirmed isolated VMs in fetuses of 20-24 weeks' gestational age, according to iuMR, were the following: normal, 2/90; mild, 66/90; moderate, 15/90; and severe, 7/90. The additional brain ab- 

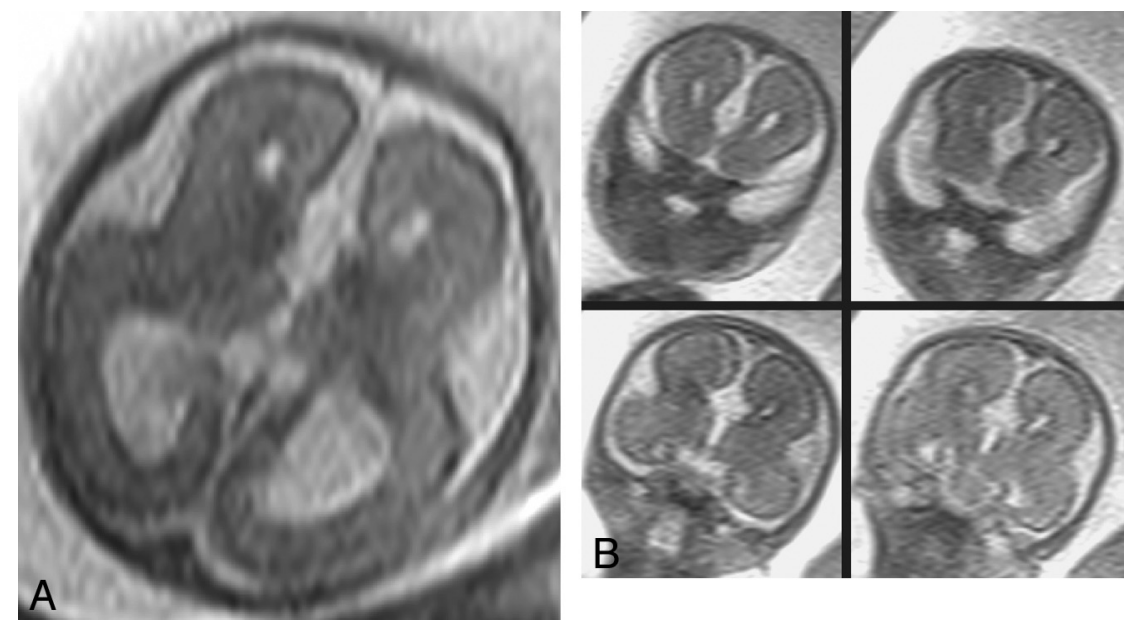

Fig 1. In utero MR imaging (iuMR) scans of a fetus at 23 weeks' gestational age thought to have isolated ventriculomegaly (VM) on sonography. $A$ and $B$, Axial $(A)$ and a montage of 4 consecutive coronal single-shot fast spin-echo images $(B)$ show agenesis of the corpus callosum with colpocephaly.
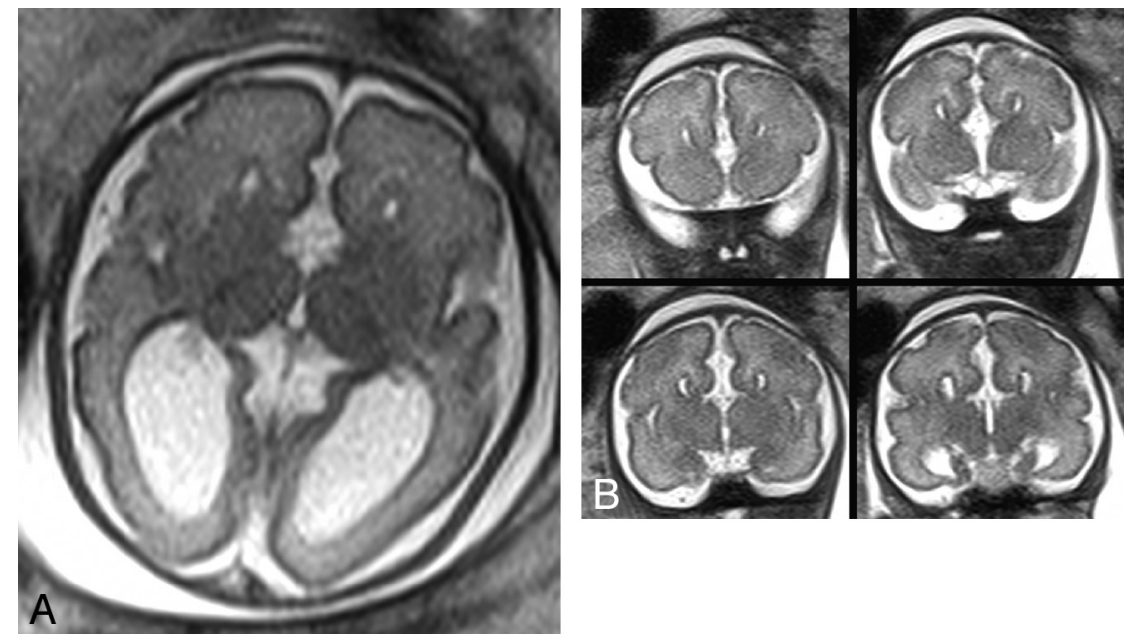

Fig 2. A fetus depicted on iuMR at 30 weeks' gestational age because of VM. $A$ and $B$, Axial $(A)$ and a montage of 4 consecutive coronal single-shot fast spin-echo images $(B)$ show agenesis of the corpus callosum with colpocephaly.

normalities shown in the 9 fetuses of this subgroup are described in on-line Table 1 and consisted of developmental abnormalities in $8 / 9$ fetuses. Failed commissuration (agenesis of the corpus callosum) was the most frequently found abnormality in fetuses with VM in the 20-24 weeks' group (5/9). An example is shown in Fig 1. The sizes of the enlarged trigones on the most severely affected side in the 9 fetuses with abnormal brains are shown in on-line Table 1 , with most cases having mild VM.

\section{Twenty-five-weeks' and Over Group}

The median gestational age range at the time of iuMR for this group was 25-27 weeks. Forty-eight fetuses were referred with isolated VM on sonography at 25 weeks' gestational age or later, most between 30 and 34 weeks. Sixteen of 48 (33\%; 95\% CI, $20 \%-48 \%$ ) fetuses had additional brain pathology. The categoric assessment of the largest trigone in the 32 cases of confirmed isolated VM according to iuMR was the following: mild, 17/32; moderate, 10/32; and severe, $5 / 32$. The details of the 16 cases with other brain abnormalities shown on iuMR are presented in on-line Table 2 and consisted of 11 developmental abnormalities and 5 acquired pathologies. As in the
$20-24$ weeks' group, failed commissuration was the most frequently associated developmental abnormality of the 25+ weeks' group (6/16, 3 associated with a neocortical abnormality). Examples are shown in Figs 2 and 3. The categories of enlarged trigones (on the most severely affected side) in the fetuses with other brain abnormalities are shown in on-line Table 2. Most fetuses had severe VM.

Table 1 shows that there is a $6 \%$ risk for fetuses with mild VM to have a further brain abnormality in both gestational age groups. The overall increase in relative risk in fetuses with severe VM was 10.3 when compared with fetuses with mild $\mathrm{VM}$, and this elevated risk did reach statistical significance.

There was excellent independent agreement between the 2 reviewers who performed the analysis on the effect on management in cases of other brain pathology being shown. There was complete agreement in 20/25 (80\%; 95\% CI, 59\%-93\%) cases, 1 grade of disagreement in 3 , and 2 grades of disagreement in 2 (on-line Tables 1 and 2). In 4/9 cases with abnormal findings in the 20-24 weeks' group, the effect of iuMR on management was judged as "iuMR gave additional information that significantly altered prognosis to a degree that termination of pregnancy was 

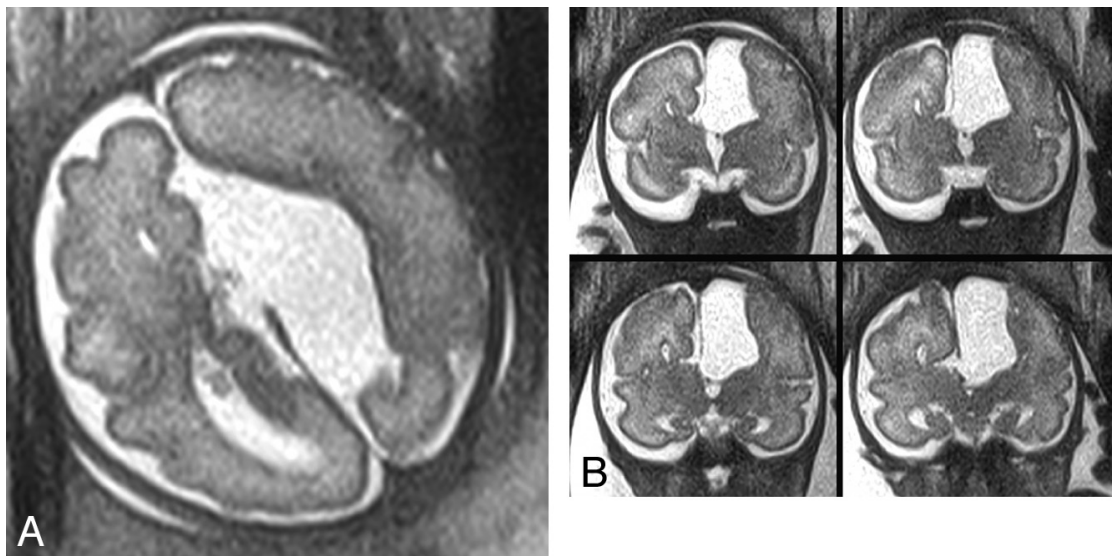

Fig 3. iuMR images of a fetus at 31 weeks' gestational age thought to have isolated VM on sonography. Axial $(A)$ and a montage of 4 consecutive coronal single-shot fast spin-echo images $(B)$ show agenesis of the corpus callosum and an associated interhemispheric cyst. The cortex adjacent to the cyst was interpreted as abnormal, but no formal confirmation of that finding is now available.

\begin{tabular}{|c|c|c|c|}
\hline \multicolumn{4}{|c|}{ Risk of detecting brain abnormalities on iuMR relative to the degree of VM of the fetus ${ }^{a}$} \\
\hline & Mild VM & Moderate VM & Severe VM \\
\hline \multirow[t]{2}{*}{ Overall risk of another brain abnormality } & $6 \%(2 \%-12 \%)$ & $14 \%(4 \%-2 \%)$ & $57 \%(37 \%-76 \%)$ \\
\hline & $5 / 90$ & $4 / 29$ & $16 / 28$ \\
\hline Overall relative risk compared with mild VM & 1 & $2.5(0.7-8.6)$ & $10.3(4.1-25.6)^{\mathrm{b}}$ \\
\hline \multirow[t]{2}{*}{ Risk of another brain abnormality in $20-24 \mathrm{w}$ group } & $6 \%(2 \%-14 \%)$ & $17 \%(4 \%-41 \%)$ & $22 \%(3 \%-60 \%)$ \\
\hline & $4 / 72$ & $3 / 18$ & $2 / 9$ \\
\hline Relative risk compared with mild VM (20-24 w) & 1 & $3.0(0.7-12.2)$ & $4.0(0.8-18.8)$ \\
\hline \multirow[t]{2}{*}{ Risk of another brain abnormality in $25 \mathrm{w}+$ group } & $6 \%(0 \%-27 \%)$ & $9 \%(0 \%-41 \%)$ & $74 \%(49 \%-91 \%)$ \\
\hline & $1 / 18$ & $1 / 11$ & $14 / 19$ \\
\hline Relative risk compared with mild VM $(25 w+)$ & 1 & $1.6(0.1-23.6)$ & $13.3(1.9-90.8)^{b}$ \\
\hline
\end{tabular}

Note:-VM indicates ventriculomegaly; w, week.

${ }^{a}$ Figures in brackets indicate $95 \%$ confidence intervals.

${ }^{b}$ Statistical significance at the $<.05 \%$ level.

offered" as judged by both reviewers. Those circumstances occurred in 2/16 cases in the $25+$ weeks' group.

\section{Discussion}

Fetal VM can arise from a number of causes, including obstruction to CSF flow (leading to hydrocephalus), focal or generalized loss of brain volume (ex-vacuo dilation), and secondary to malformation of the CNS (dysmorphic). In many cases, however, no cause is found either before or after delivery. The 2 clinical considerations in a fetus with VM are the degree of ventricular enlargement and the presence or absence of other abnormalities, of which CNS abnormalities are common. It is widely accepted that severe categories of VM are associated with higher risks of poor clinical outcome, ${ }^{12}$ and indeed recent studies have questioned whether mild VM (trigone measurements of $10-12 \mathrm{~mm}$ ) is truly abnormal if it is an isolated finding. ${ }^{13,14}$ For example, in a study of 176 cases, Gaglioti et $\mathrm{al}^{7}$ showed that a fetus with isolated VM had a $97.7 \%$ chance of being alive at 2 years if the VM was mild, an $80 \%$ chance with moderate VM, and a 33.3\% chance with severe VM. Of those that were alive, neurodevelopmental outcome was normal in $93 \%$ of mild cases, $75 \%$ of moderate, and $62.5 \%$ of severe VM cases.

An earlier study by Gupta et $\mathrm{al}^{15}$ described 276 cases of apparent isolated VM, all of whom were delivered. They found a $70 \%$ survival rate, and 59\% of the survivors were developmentally normal. Most centers referring cases into our study quote figures of $<5 \%$ chance of adverse neurologic outcome in cases of mild VM if there are no other CNS abnormalities. This view has been supported in the recent paper of Falip et $\mathrm{al},{ }^{10}$ who incorporated iuMR study results into their antenatal definition of isolated VM. They showed that the outcome of isolated VM was excellent in fetuses with 10- to $11.9-\mathrm{mm}$ trigones in $94 \%$ of cases and in $85 \%$ of cases with trigone measurements of $12-15 \mathrm{~mm}$.

There have been improvements in sonographic image quality which, together with an increased understanding of the importance of failed commissuration (eg, agenesis of the corpus callosum) in prognosis, have contributed to improved detection of isolated VM. The presence of other CNS abnormalities is important because it is the brain abnormality that is responsible for the postnatal morbidity and mortality in most cases rather than VM per se. ${ }^{16}$ It is of major importance, therefore, to have sought other pathology with the best imaging method available.

The introduction of iuMR into clinical practice has provided a supplementary method of assessing the fetal brain. There could be no a priori reason to believe that iuMR would be more accurate than sonography in assessing the size of fetal ventricles. Early work by Garel and Alberti ${ }^{17}$ seemed to support that assumption, but a more recent publication, including the same author, showed the opposite conclusion. ${ }^{18}$ The retrospective analysis of Salomon et $\mathrm{al}^{18}$ included 185 thirdtrimester fetuses who had isolated mild VM (ie, 10- to 12-mm trigones) as measured on sonography. They found that the most frequent disagreement between sonography and iuMR 
was in the classification of the degree of VM. Forty-three fetuses $(23.3 \%$ ) had ventricles $<10 \mathrm{~mm}$ on iuMR (ie, normal size), and 36 fetuses (19.4\%) had trigone measurements of $>12 \mathrm{~mm}$ on iuMR. All of those findings would have had significant effects on the information given to the parents.

The work described in our article supports the authors' original findings - that is, there is little disagreement between the dimensions of the trigones measured on sonography when compared with iuMR. In the 122 cases of isolated VM confirmed by iuMR in this study, there was complete categoric agreement about the degree of VM in approximately $90 \%$. In all but 1 case of categoric disagreement, there was a difference in absolute measurements of no greater than $2 \mathrm{~mm}$, which is in broad agreement with the work of Salomon et al. ${ }^{18}$ We found only 2 cases diagnosed on sonography as mild VM with trigone measurements of $<10 \mathrm{~mm}$ on iuMR.

On the basis of those findings, we conclude that iuMR does not have a role in assessing the size of the ventricular trigones over and above sonography, but with 2 caveats. There are sometimes problems in visualizing the entire ventricular system on sonography because of factors such as fetal position and problems caused by calvarial ossification. ${ }^{19}$ There is also a specific problem in visualizing the lateral ventricle closest to the sonography probe (near-field effect), which may be of relevance in cases of asymmetric VM. We have not addressed the near-field effect in the current work, but our earlier work suggested that fetal ventricular morphology is an important indicator of brain malformation. ${ }^{20}$

There is growing evidence that iuMR has a higher detection rate of fetal brain abnormalities when compared with sonography. ${ }^{21-23}$ Our earlier study of 100 fetuses showed an overall improvement in diagnostic accuracy of $48 \%$ for unselected cases of fetal CNS abnormalities. ${ }^{24}$ Fetuses with VM as part of their referral information accounted for approximately half of those cases, and an improvement in the detection rate of close to $50 \%$ was found in that subgroup. With particular reference to fetal VM, Launay et $\mathrm{al}^{25}$ concluded that iuMR was "more informative than sonography in $32.8 \%$ of cases" and showed the cause of the VM in $21.3 \%$ of cases in a study of 61 fetuses. As described earlier, Salomon et al ${ }^{18}$ studied 185 third-trimester fetuses with isolated mild VM. With the exception of $43 \%$ of cases in which there was a disagreement about the category of VM, 11/185 (5.9\%) had other brain abnormalities. Our earlier work, based on a study of the first 30 fetuses with isolated VM referred between 1999 and 2003, showed that iuMR depicted additional abnormalities in $50 \%{ }^{26}$

Selection bias is a major problem in interpreting such studies, particularly retrospective reviews of clinical referrals, and our present prospective evaluation of fetuses with isolated VM provided an opportunity to control the referral bias as much as possible. In particular, we started with the assumption that the strict criteria for inclusion into the study would be likely to provide the minimum improvement in diagnostic accuracy of any group with fetal CNS abnormalities shown on sonography. If that is true, the overall improvement in detection of $17 \%$ must be considered as highly relevant. All of the obstetricians and radiologists from our referring centers were free to enroll all of their cases of isolated VM. However, we did not study how many women were not asked to join the study, nor do we know how many were asked and refused. We must accept, therefore, that we have not eliminated referral bias, and we do not know the depth of the problem. For example, it is possible that the fetomaternal experts were more likely to refer fetuses with severe VM because they had believed that iuMR was more likely to find a further brain abnormality.

Before we discuss our results, it is important to highlight a weakness of the study as it stands. Ideally a study such as this should have a reference standard diagnosis made by follow-up postnatal imaging or autopsy studies, but this has not been performed as yet. Our earlier studies have provided that level of detail, and iuMR was always as good as the reference standard, ${ }^{11}$ but we do not have those data for the cases in this study at present. In defense of that, many of the diagnoses made in this study could be considered "true by definition" (eg, agenesis of the corpus callosum, intracranial hemorrhage) as is the case in pediatric neuroimaging. We have to accept, however, that the diagnoses of "abnormalities of cortical formation," for example, cannot be taken as read at present. It is our intention to perform clinical and radiologic studies later in life on the children born in this study. Due comment must also be made about the problems of obtaining postmortem information for studies performed in the United Kingdom because of the very low autopsy rate as discussed previously by Griffiths et al. ${ }^{27}$

Our results show that there is a clear relationship between the degree of VM and the risk of other brain abnormalities. In the entire group of fetuses studied, severe VM was approximately 10 times more likely to be found in association with a brain abnormality than mild VM. iuMR was more likely to find a brain malformation in the $25+$ weeks' group when compared with the 20-24 weeks' group, though this is probably explained by the higher frequency of severe VM cases in the later gestational age group. The small number of fetuses recruited into the severe VM group makes interpretation problematic, but as the data stand, the relative risk of a brain abnormality with severe VM in the $25+$ weeks' group was much higher (13.3) than in the 20-24 weeks' group (4.0).

There was, however, a $6 \%$ chance of showing a brain abnormality in cases of mild VM at any gestational age older than 20 weeks. A wide range of brain pathology was shown by iuMR, both developmental and acquired. One malformation that was featured most frequently was failed commissuration (agenesis of the corpus callosum), which accounted for 11/25 (44\%) cases either alone or in conjunction with further brain abnormalities. This suggests that failed commissuration can be a difficult diagnosis on sonography. Why that structure presents such difficulties is a subject of debate and warrants further investigation. In many cases, it is difficult or impossible to visualize the corpus callosum directly in the fetus with sonography, and the cavum septum pellucidum is often used as a surrogate indicator that the corpus callosum is present. It has also been raised recently whether there is a potential for misidentification of the septal leaves because of abnormally placed fornices. ${ }^{28-30}$ The role of advanced sonographic methods such as $3 \mathrm{D} / 4 \mathrm{D}$ sonography with its ability to create reconstructed sonographic images in the midline is also under evaluation.

The detection of CNS abnormalities by iuMR has been shown to have a high impact on clinical management in other studies, but not specifically in isolated VM cases. Simon et $\mathrm{al}^{22}$ showed that $46 \%$ of 52 cases were managed differently after 
iuMR, and a similar analysis made by Levine et $\mathrm{al}^{31}$ showed an overall $13.5 \%$ rate of management change and a counseling change in $49.7 \%$. Those authors have suggested that in fetuses with VM "MR can be helpful in visualizing associated abnormalities...," but they did not say which cases should be selected for iuMR. ${ }^{9}$ Significant effects on clinical management in our study occurred most frequently in the 20-24 weeks' group of pregnancies; the assessors judged that termination of pregnancy would have been considered in 4/9 cases. Similar changes in patient management were less likely in the $25+$ weeks' group, occurring in only $2 / 16$. The most likely explanation for this is the severity of VM associated with the brain malformation. The negative effect on outcome for the more severe forms of VM has been redemonstrated by Ouahba et $\mathrm{al}^{32}$ recently in a study that included iuMR investigations to confirm or refute isolated VM. Severe VM was much more common in the 25+ weeks' group, and the assessors judged that women with fetuses with severe VM would have been advised of high risks of poor outcomes and perhaps offered termination of pregnancy irrespective of the information about brain malformation provided by iuMR.

This point is well illustrated in our 20-24 weeks' group, in which $3 / 5$ of the cases of failed commissuration were associated with mild or moderate VM, and termination of pregnancy would have been considered as a result of the iuMR. In contrast, in the 2 cases in the 20-24 weeks' group with failed commissuration and severe $\mathrm{VM}$, the effect on management brought about by the detection of the additional brain malformation was minor.

\section{Conclusions}

Our work demonstrates that there is a good case for including iuMR as part of the diagnostic process for fetuses thought to have isolated VM on sonography at any time after 20 weeks' gestational age, with additional brain abnormalities shown in $17 \%$. It could be argued that any resource to provide iuMR on a national basis is best directed at the 20-24 weeks' group. Although the improvement in detection rate was only $6 \%$ in that group, there was a higher impact on clinical management as judged by the independent assessors. It should be noted, however, that some significant changes in clinical management were brought about by the inclusion of iuMR in the older gestational age fetuses.

\section{References}

1. Cardoza JD, Goldstein RB, Filly RA. Exclusion of fetal ventriculomegaly with a single measurement: the width of the lateral ventricular atrium. Radiology 1988;169:711-14

2. Heiserman J, Filly RA, Goldstein RB. Effect of measurement errors on sonographic evaluation of ventriculomegaly. J Ultrasound Med 1991;10:121-24

3. Twickler DM, Reichel T, McIntire DD, et al. Fetal CNS ventricle and cisterna magna measurements by magnetic resonance imaging. Am J Obstet Gynecol 2002;187:927-31

4. Nicolaides $\mathrm{KH}$, Gosden CM, Snijders RJM. Ultrasonographically detectable markers of fetal chromosomal defects. In: Nelson JP, Chambers SE, eds. Obstetric Ultrasound: Volume 1. Oxford, UK: Oxford University Press; 1993:41-82

5. Nicolaides KH, Berry S, Snijders RJ, et al. Fetal lateral cerebral ventriculomegaly: associated malformations and chromosomal defects. Fetal Diagn Ther 1990;5:5-14

6. Nyberg DA, Mack LA, Hirsch J, et al. Fetal hydrocephalus: sonographic detection and clinical significance of associated abnormalities. Radiology 1987; 163:187-91

7. Gaglioti P, Danelon D, Bontempo S, et al. Fetal cerebral ventriculomegaly: outcome in 176 cases. Ultrasound Obstet Gynecol 2005;25:372-77

8. Filly RA, Cardoza JD, Goldstein RB, et al. Detection of fetal CNS anomalies: a practical level of effort for a routine sonogram. Radiology 1989;172:403-08

9. Mehta TS, Levine D. Imaging of fetal cerebral ventriculomegaly: a guide to management and outcome. Semin Fetal Neonatal Med 2005;10:421-28

10. Falip C, Blanc N, Maes E, et al. Postnatal clinical and imaging follow-up of infants with prenatal isolated mild ventriculomegaly: a series of 101 cases. Pediatr Radiol 2007;37:981-89

11. Whitby E, Paley MN, Davies $\mathrm{N}$, et al. Ultrafast magnetic resonance imaging of central nervous system abnormalities in utero in the second and third trimester of pregnancy: comparison with ultrasound. BJOG 2001;108:519-26

12. Graham E, Duhl A, Ural S, et al. The degree of antenatal ventriculomegaly is related to pediatric neurological morbidity. J Matern Fetal Med 2001;10: $258-63$

13. Filkins K, Koos BJ. Ultrasound and fetal diagnosis. Curr Opin Obstet Gynecol 2005; 17:185-95

14. Davis GH. Fetal hydrocephalus. Clin Perinatol 2003;30:531-39

15. Gupta JK, Bryce FC, Lilford RJ. Management of apparently isolated fetal ventriculomegaly. Obstet Gynecol Surv 1994;49:716-721

16. Filly RA, Goldstein RB, Callen PW. Fetal ventricle: importance in routine obstetric ultrasonography. Radiology 1991;181:1-7

17. Garel C, Alberti C. Coronal measurement of the fetal lateral ventricles: comparison between ultrasonography and magnetic resonance imaging. Ultrasound Obstet Gynecol 2006;27:23-27

18. Salomon LJ, Ouahba J, Delezoide AL, et al. Third trimester fetal MRI in isolated 10- to 12-mm ventriculomegaly: is it worth it? BJOG 2006;113:942-47. Epub 2006 Jul 7

19. Golja AM, Estroff JA, Robertson RL. Fetal imaging of CNS abnormalities. Neuroimag Clin N Am 2004;14:293-306

20. Rickard S, Morris J, Paley M, et al. In utero magnetic resonance of non-isolated ventriculomegaly: does ventricular size or morphology reflect pathology. Clin Radiol 2006;61:844-53

21. Levine D, Barnes PD, Madsen JR, et al. Fetal CNS anomalies: MR imaging augments sonographic diagnosis. Radiology 2004;204:635-42

22. Simon EM, Goldstein EB, Coakley FV, et al. Fast MR imaging of fetal CNS anomalies in utero. AJNR Am J Neuroradiol 2000;21:1688-98

23. Griffiths PD, Paley MNJ, Widjaja E, et al. The emergence of in utero MR imaging for fetal brain and spine abnormalities. BMJ 2005;331:562-65

24. Whitby EH, Paley MNJ, Sprigg A, et al. Outcome of 100 singleton pregnancies with suspected brain abnormalities diagnosed on ultrasound and investigated by in utero MR imaging. BJOG 2004;111:784-92

25. Launay S, Robert Y, Valat AS, et al. Cerebral fetal MRI and ventriculomegaly. J Radiol 2002;83:723-30

26. Morris JE, Rickard S, Paley MN, et al. The value of in utero MR imaging in ultrasound diagnosed foetal isolated cerebral ventriculomegaly. Clin Radiol 2007;62:140-44

27. Griffiths PD, Variend D, Evans M, et al. Postmortem magnetic resonance imaging of the fetal and stillborn central nervous system. AJNR Am J Neuroradiol 2003;24:22-27

28. Griffiths PD, Batty R, Reeves MJ, et al. Imaging the corpus callosum, septum pellucidum and fornix in children: normal anatomy and variations of normality. Neuroradiology 2009;51:337-45

29. Griffiths PD, Batty R, Connolly DA, et al. Effects of failed commissuration on the septum pellucidum and fornix: implications for fetal imaging. Neuroradiology 2009;51:347-56. Epub 2009 Mar 10

30. Callen PW, Callen AW, Glenn OA, et al. Columns of the fornix, not to be mistaken for the cavum septi pellucidi on prenatal sonography. J Ultrasound Med 2008;279:25-31

31. Levine D, Barnes PD, Robertson RR, et al. Fast MR imaging of fetal CNS abnormalities. Radiology 2003;229:51-61

32. Ouahba J, Luton D, Vuillard E, et al. Prenatal isolated mild ventriculomegaly: outcome in 167 cases. BJOG 2006;113:1072-79 